\title{
Adesão ao tratamento de adolescentes com diabetes mellitus tipo 1
}

\author{
Adherence to treatment of adolescents with type 1 diabetes mellitus
}

\author{
Renata Simionato ${ }^{a}$, Tayla Karolina da Rochab ${ }^{b}$ Amanda Aires Lombardinib, Karin Viegasc, \\ Gisele Pereira de Carvalho ${ }^{d}$, Simone Travi Canabarro ${ }^{\mathrm{e}}$

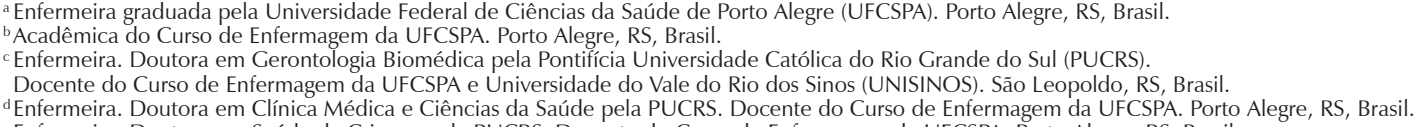

RESUMO

Introdução: O diabetes mellitus (DM) é uma das mais importantes doenças crônicas da atualidade, acometendo milhões de pessoas em todo mundo. O controle efetivo da doença é indispensável para evitar complicações futuras. Para isso, é necessária uma dieta balanceada, prática de atividade física regular, monitorização da glicemia capilar e insulinoterapia.

Objetivo: Identificar os aspectos determinantes de adesão ao tratamento de adolescentes com DM1, atendidos em um centro de referência em DM do sul do Brasil.

Materiais e Métodos: Estudo transversal, descritivo e analítico, realizado com 45 adolescentes diagnosticados com DM1. A coleta de dados foi realizada em um centro de referência em DM, por meio de um questionário sociodemográfico e aplicação de escalas para a avaliação do autocuidado e adesão ao tratamento. Os dados foram analisados no SPSS ${ }^{\circledR}$ versão 20.0 para Windows. O presente estudo foi aprovado pelos Comitês de Ética e Pesquisa das instituições envolvidas.

Resultados: Prevaleceram meninos entre 10-19 anos, estudantes e de etnia branca. Na análise da hemoglobina glicada, 40 participantes (88,9\%) apresentaram valores acima do esperado, $25(55,5 \%)$ tiveram escore para provável adesão ao tratamento e 34 (75,5\%) apresentaram déficit moderado de motivação.

Conclusão: Observou discordância entre os valores de hemoglobina glicada e adesão ao tratamento, sendo que os fatores que contribuem para tal referem-se ao esquema terapêutico de múltiplas doses de insulina e à dificuldade que os pacientes possuem quanto a sua aplicação, destacando-se a baixa motivação às recomendações de vida saudável.

Palavras-chave: doenças crônicas; autocuidado; comportamento do adolescente.

Introduction: Diabetes mellitus (DM) is one of the most important chronic diseases, affecting millions of people around the world. The effective control of the disease is indispensable to avoid future complications. For this, a balanced diet, practice of regular physical activity, monitoring of capillary glycemia and insulin therapy are necessary.

Objective: To identify the determinants of adherence to treatment of adolescents with DM1, attended at a reference center in DM in southern Brazil.

Materials and Methods: A cross-sectional, descriptive and analytical study was carried out with 45 adolescents diagnosed with DM1. The data collection was performed at a reference center in DM, through a sociodemographic questionnaire and application of scales for the evaluation of self-care and adherence to treatment. The data were analyzed in SPSS ${ }^{\circledR}$ version 20.0 for Windows. The present study was approved by the Ethics and Research Committees of the institutions involved.

Results: Boys aged 10-19 years old, students and white ethnicity prevailed. In the analysis of glycated hemoglobin, 40 participants (88.9\%) had values higher than expected, 25 (55.5\%) had a score for probable treatment adherence and 34 (75.5\%) presented moderate motivation deficit.

Conclusion: It observed a disagreement between the values of glycated hemoglobin and adherence to the treatment, and the factors that contribute to this refer to the therapeutic regimen of multiple doses of insulin and the difficulty that the patients have regarding their application, standing out the low motivation for the healthy living recommendations.

Keywords: chronic disease; self care; adolescent behavior. 


\section{INTRODUÇÃO}

O diabetes mellitus tipo 1 (DM1), também denominado como diabetes juvenil e/ou insulino dependente, é responsável por cerca de $10 \%$ dos casos de diabetes no Brasil. Neste caso, ocorre a destruição da célula beta do pâncreas, devido a um processo autoimune ou de outras doenças degenerativas que se associam ao DM1, comprometendo a capacidade total de produção de insulina que é necessária para baixar os níveis glicêmicos e assim prevenir a cetoacidose, coma e a morte ${ }^{1}$.

Pacientes com DM1 dependem do uso de múltiplas doses de insulina diariamente e são mais propensos a ter quadros de cetoacidose. De acordo com estimativas, 2 a 3\% dos pacientes com DM1 morrem dentro de 10 anos após o diagnóstico, e 12 a 13\% morrem 20 anos após o diagnóstico decorrente de complicações agudas ou crônicas. A identificação do DM na infância leva a um grande impacto emocional na família, fazendo com que, muitas vezes, haja uma negação em relação ao diagnóstico e objeção ao tratamento ${ }^{2,3}$. Diante disso, é possível afirmar que para a adesão ao tratamento é imprescindível conhecer as necessidades sociais e psicossociais do paciente.

Segundo a Organização Mundial da Saúde (OMS), a adesão ao tratamento é o grau de correspondência e concordância do paciente com as recomendações do médico ou de outro profissional da saúde no que se refere à ingestão de medicamentos, seguimento da dieta e mudanças nos hábitos de vida.

Nesse sentido, os adolescentes compõem o grupo mais suscetível aos agravos do DM1, visto que a adesão ao tratamento se torna ainda mais difícil em virtude da singularidade deste período de transição. Com a doença, surgem limitações e novas responsabilidades que podem interferir no desenvolvimento desse jovem. A luta pela independência em relação aos pais pode levar a baixa adesão ao regime terapêutico do tratamento. Além disso, algumas dificuldades em manter o autocuidado, como manter uma dieta adequada, monitorar a glicemia regularmente e realizar a aplicação da insulina, podem levar, além de uma descompensação metabólica, ao isolamento social e a uma má relação com os pais ${ }^{4,5}$.

A baixa aderência ao tratamento pode levar a exacerbação da doença, e é um dos fatores mais importantes para o mau controle da doença, podendo levar, alguns casos, a mortalidade precoce, além do aumento de custos para o sistema de saúde. No ano de 2015, estima-se que 5 milhões de adultos morreram, em todo o mundo, devido ao diabetes ${ }^{6}$.

Diante do fato de que a baixa adesão ao tratamento de DM pode trazer efeitos prejudiciais em longo prazo $^{5}$, justifica-se a importância deste estudo, sobre os aspectos determinantes de adesão ao tratamento de adolescentes e adultos jovens com DM1, para subsidiar discussões e formular intervenções que estimulem o autocuidado e a adesão ao tratamento, diminuindo o risco de complicações agudas e crônicas do diabetes e promovendo melhora na qualidade de vida dessa população.

Dessa forma, é objetivo desse trabalho identificar os aspectos determinantes de adesão ao tratamento de adolescentes e adultos jovens com DM1 atendidos em um Centro de Referência em DM do sul do Brasil, traçar o perfil sócio demográfico dessa população e avaliar a motivação para o autocuidado desses pacientes.

\section{MATERIAIS E MÉTODOS}

Trata-se de um estudo transversal, descritivo e analítico, coletado prospectivamente e desenvolvido no município de Porto Alegre, Rio Grande do Sul, em um centro de referência para crianças e adolescentes com diabetes mellitus.

O público alvo foi constituído por 45 adolescentes atendidos no centro de referência no período entre abril a maio de 2016. A amostra do estudo foi intencional e os participantes foram selecionados de forma aleatória, por conveniência, e intencional enquanto aguardavam a consulta, seguindo os seguintes critérios de inclusão: diagnóstico clínico de DM1; idade na faixa etária de 10 a 24 anos; tempo de diagnóstico superior a 2 anos; frequentar o centro de referência nos últimos 6 meses, aceitar participar da pesquisa e assinar o Termo de Consentimento Livre e Esclarecido. Para os participantes com idade inferior a 18 anos, os responsáveis tiveram que assinar o Termo de Consentimento e os participantes o Termo de Assentimento.

A coleta de dados deu-se por meio da aplicação de entrevistas semiestruturadas realizadas na própria Instituição, duas vezes por semana, no turno da manhã, em uma sala reservada, destinada para a mesma. As entrevistas individuais transcorreram em aproximadamente 20 minutos de duração, sendo realizadas, em média, 4 entrevistas por dia, onde realizava-se a aplicação de dois questionários.

No que diz respeito aos instrumentos de coleta, fez-se uso de um questionário para investigar os dados sociodemográfico, histórico familiar e condições de saúde dos participantes construído pelos próprios pesquisadores. Quanto a avaliação do autocuidado, foi utilizada subescala III da Escala para Determinação da Competência do Diabético para o Autocuidado (ECDAC), que determina as capacidades motivacionais e emocionais para o autocuidado, relacionada em 11 itens que abordam autoestima, autovalorização, controle emocional para fazer julgamentos e executar ações, autodisciplina e aceitação de sua situação de pessoa portadora de diabetes. Para a avaliação da adesão ao tratamento foi 
aplicado o Teste de Brief Medication Questionaire (BMQ), validado no Brasil em 2012 por Ben, Neumann e Mengue. É um instrumento dividido em três domínios que buscam identificar barreiras à adesão quanto ao regime, às crenças e à recordação em relação ao tratamento medicamentoso na perspectiva do paciente ${ }^{7}$.

Os dados foram analisados por estatística descritiva de acordo com os registros dos questionários e dos relatos dos adolescentes. Os dados quantitativos coletados foram armazenados em Microsoft Exce ${ }^{\circledR} \mathrm{e}$, posteriormente, transcritos e analisados no software SPSS ${ }^{\circledR}$ versão 20.0 para Windows. As variáveis foram descritas por frequências, médias e desvios padrões. As variáveis do BMQ (regime, crenças, recordação e total) foram avaliadas por correlação não paramétrica (R2), e após foi realizada a regressão linear múltipla nas correlações que se apresentaram significativas $(p=0,05)$.

Todos os princípios éticos foram respeitados em relação ao acesso e à análise dos dados, atendendo à Resolução no 466/12 do Conselho Nacional de Saúde. A pesquisa foi submetida a análise e aprovação das Comissões de Ética e Pesquisa da Universidade Federal de Ciências da Saúde de Porto Alegre e do Comitê de Ética e Pesquisa do Grupo Hospitalar Conceição, e aprovados sob os pareceres de número 1.401 .990 e 1.434.607, respectivamente.

\section{RESULTADOS}

Dos $45(62,2 \%)$ participantes, houve predominância do sexo masculino, 33 (73,3\%) apresentavam idade entre 10 e 19 anos e 12 (26,7\%) entre 20 e 24 anos, 36 (80\%) autodeclaravam-se da cor branca. Quanto a ocupação, 32 $(73,3 \%)$ eram estudantes, 35 (77,9\%) não possuíam renda própria, e $20(44,4 \%)$ estavam no ensino médio (Tabela 1).

Em referência às condições de saúde dos participantes, a partir da análise do índice de Massa Corporal (IMC) ou Z-escore (aplicado para os menores de 20 anos), verificouse que $33(73,3 \%)$ apresentavam peso adequado para a idade, enquanto $11(24,4 \%)$ estavam com sobrepeso. Quando foi perguntado aos participantes sobre como eles consideravam a própria condição de saúde e a visão, 20 $(44,4 \%)$ consideraram possuir uma saúde boa ou regular e 19 $(42,2 \%)$ ter uma visão boa. Em relação ao fumo, 41 (91,1\%) dos participantes não fumam e $14(31,1 \%)$ residem com pelo menos uma pessoa fumante.

A história de comorbidades dos participantes foi baseada de acordo com o relato dos entrevistados ou de seus familiares. Na faixa etária de 10 a 19 anos, $2(4,4 \%)$ dos participantes apresentavam doença respiratória crônica, $1(2,2 \%)$ tuberculose. Na faixa etária de 20 a 24 anos, 2 $(4,4 \%)$ dos participantes apresentavam Hipertensão Arterial Sistêmica (HAS), 1 (2,2\%) retinopatia e 1 (2,2\%) depressão.
Tabela 1. Distribuição socioeconômica dos adolescentes e adultos jovens participantes do estudo.

\begin{tabular}{|c|c|c|}
\hline Variáveis & Frequência & $\%$ \\
\hline \multicolumn{3}{|l|}{ Sexo } \\
\hline Masculino & 28 & 62,2 \\
\hline Feminino & 17 & 37,8 \\
\hline \multicolumn{3}{|l|}{ Faixa Etária } \\
\hline 10 a 19 anos & 33 & 73,3 \\
\hline 20 a 24 anos & 12 & 26,7 \\
\hline \multicolumn{3}{|l|}{ Raça } \\
\hline Branca & 36 & 80 \\
\hline Preta & 4 & 8,9 \\
\hline Parda & 5 & 11,1 \\
\hline \multicolumn{3}{|l|}{ Renda familiar (em salários mínimos) } \\
\hline Não possui renda própria & 35 & 77,9 \\
\hline$<1 \mathrm{SM}$ & 2 & 4,4 \\
\hline$\geq 1 \mathrm{a}<2 \mathrm{SM}$ & 5 & 11,1 \\
\hline$\geq 2 \mathrm{a}<4 \mathrm{SM}$ & 2 & 4,4 \\
\hline$\geq 4 \mathrm{a}<5 \mathrm{SM}$ & 1 & 2,2 \\
\hline \multicolumn{3}{|l|}{ Grau de instrução } \\
\hline Primário completo/Fundamental incompleto & 12 & 26,7 \\
\hline Fundamental completo/Médio incompleto & 20 & 44,4 \\
\hline Médio completo & 7 & 15,6 \\
\hline Superior incompleto & 5 & 11,1 \\
\hline Superior completo & 1 & 2,2 \\
\hline \multicolumn{3}{|l|}{ Ocupação } \\
\hline Estudante & 33 & 73,3 \\
\hline Trabalho remunerado & 9 & 20 \\
\hline Não respondeu & 3 & 6,7 \\
\hline
\end{tabular}

A análise do último registro do exame da hemoglobina glicada (HbA1c) mostrou que 40 (88,9\%) dos entrevistados possuíam risco de complicações crônicas e que apenas $5(11,1 \%)$ possuíam bom controle glicêmico. Sobre as internações e hipoglicemias, 35 (77,8\%) não tiveram internações hospitalares e nem hipoglicemias graves no último ano. Quanto à frequência das consultas, 39 (86,7\%) dos indivíduos frequentam a Instituição a cada 3 ou 4 meses. Quanto ao tempo de diagnóstico de diabetes, 33 $(73,3 \%)$ dos participantes possuíam o diagnóstico de DM1 a mais de 5 anos. No que diz respeito a história familiar dos adolescentes e adultos jovens com DM1, 22 (48,9\%) das mães dos entrevistados não apresentavam comorbidades, $9(20 \%)$ tinham doença coronariana e $4(8,9 \%)$ possuíam DM. Em relação aos pais, 36 (80\%) deles não apresentavam comorbidades e $1(2,2 \%)$ possuía DM.

Foi feita a correlação entre as variáveis BMQ (regime, crenças, recordação e total), sendo que o coeficiente foi significativo entre o $B M Q$ regime e $B M Q$ crenças $\left(R^{2}=0,549\right.$; $p<0,0001)$, BMQ regime e BMQ total $\left(R^{2}=-0,667\right.$; $p<0,0001)$ e $B M Q$ crenças e BMQ total $\left(R^{2}=-0,454\right.$; $p=0,002)$. Dos coeficientes significativos, foram selecionadas as variáveis $\mathrm{BMQ}$ regime e $\mathrm{BMQ}$ crenças para a construção da regressão linear múltipla, identificando quais das variáveis 
que influencia no escore total. A variável $B M Q$ regime da equação explica $50 \%$ da variabilidade do BMQ total $\left(R^{2}=0,5\right)$.

Em relação aos fatores relacionados ao tratamento medicamentoso, o teste Brief Medication Questionnaires, avaliou a adesão dos pacientes ao tratamento, demonstrando que $25(55,5 \%)$ dos pacientes obtiveram um escore compatível com provável adesão. Para o domínio "Regime", que aborda as dificuldades do paciente em listar as medicações, 34 (75,5\%) não apresentaram barreiras neste estudo (escore 0). No domínio relacionado às "Crenças", que diz respeito à presença ou não de medicamentos que não funcionam bem, ou que causam algum problema para o paciente, $41(91,1 \%)$ dos pacientes não apresentaram barreiras (escore 0). No domínio "Recordação", os 45 participantes obtiveram um escore compatível com não adesão (escore $\geq 1$ ).

Por fim, com relação aos dados referentes aos fatores relacionados à motivação para o autocuidado, a aplicação do ECDAC demonstrou que $34(75,5 \%)$ dos participantes apresentaram déficit moderado de motivação para o autocuidado, $8(17,8 \%)$ apresentaram déficit severo para o autocuidado enquanto apenas $3(6,7 \%)$ apresentaram comportamento motivacional positivo para o autocuidado (Tabela 2).

Tabela 2. Capacidades motivacionais e emocionais para o autocuidado dos participantes.

\begin{tabular}{lc}
\hline ECDAC - Subescala III & n (\% \\
\hline Comportamento motivacional positivo para o autocuidado & $3(6,7)$ \\
Déficit moderado de motivação para o autocuidado & $34(75,5$ \\
Déficit severo de motivação para o autocuidado & $8(17,8)$ \\
Nenhuma motivação para o autocuidado & $0(0,0)$ \\
\hline
\end{tabular}

\section{DISCUSSÃO}

No presente estudo, em relação ao gênero, a amostra apresentou uma prevalência do sexo masculino em $62,2 \%$. No entanto, não há uma estatística nacional entre as idades de 0 a 20 anos que comprove ou não associação do DM1 com o sexo ${ }^{8}$.

Quanto à análise do IMC ou z-escore apresentou uma prevalência de $24,4 \%$ dos participantes com sobrepeso; desses, 81,8\% apresentaram controle glicêmico inadequado. Outro estudo, realizado em Goiânia, obteve uma prevalência de excesso de peso de 14,1\% quanto ao IMC/idade; desses pacientes, 90,0\% apresentaram controle glicêmico inadequado ${ }^{9}$.
Quanto aos resultados da condição de saúde e da visão, $44,4 \%$ consideraram possuir uma saúde boa ou regular e $42,2 \%$ consideraram ter uma visão boa. No que diz respeito às comorbidades, doença coronariana, HAS, e DM foram as que tiveram maior prevalência no histórico familiar dos participantes do estudo. Isso aponta para a necessidade de uma abordagem de cuidado da equipe de saúde, pois essas doenças possivelmente serão causas de agravos num futuro próximo para essa população. O que corrobora com os resultados glicêmicos encontrados que demostram que $88,9 \%$ tem riscos de complicações crônicas.

A medida da HbA1c é um método que possibilita avaliação do controle glicêmico em longo prazo e deve ser solicitada rotineiramente a todos pacientes com DM de duas a quatro vezes ao ano. Assim, consegue-se avaliar a média das glicemias do paciente nos últimos 120 dias. A recomendação da American Diabetes Association (ADA) para o controle glicêmico de crianças e adolescentes, de todas as faixas etárias, é que a $\mathrm{HbA} 1 \mathrm{c}$ se mantenha menor que $7,5 \%$, enquanto que nos adultos o ideal é que a $\mathrm{HbA} 1 \mathrm{c}$ permaneça menor que $7 \%{ }^{10}$.

O índice de HbA1c para avaliar o controle glicêmico dos participantes teve um resultado significativo neste estudo. A análise mostrou que $88,9 \%$ dos entrevistados possuíam resultados glicêmicos acima do esperado e que apenas $11,1 \%$ possuíam bom controle glicêmico, conforme parâmetros atuais ADA. Outro estudo realizado em 2013, obteve resultados semelhantes quanto a HbA1c, 80,9\% dos pacientes apresentaram valores de HbA1c acima dos valores de referência e apenas 19,1\% tinham valores dentro da taxa esperada. Além disso, foi observada associação do controle glicêmico com as variáveis hábitos alimentares, condição socioeconômica e estado afetivo-emocional ${ }^{11}$.

Diante do fato de que a redução dos níveis de HbA1c para valores $<7 \%$ diminui as complicações microvasculares e neuropáticas e, possivelmente, as complicações macro vasculares do diabetes, sobretudo no DM1 ${ }^{12}$, as taxas elevadas de valores glicêmicos acima do esperado evidenciam a dificuldade de os participantes aderirem ao tratamento, seja pelo modelo terapêutico de inúmeras doses de insulina por dia, ou pela baixa motivação em manter uma vida com hábitos saudáveis.

No entanto, os resultados do BMQ total, que avaliou a adesão dos pacientes ao tratamento medicamentoso, mostraram que 55,5\% dos participantes obtiveram um escore compatível com provável adesão, 40\% provável baixa adesão, 4,4\% baixa adesão, nenhum participante apresentou escore de adesão plena ao tratamento. Em um estudo realizado em Ribeirão Preto-São Paulo, no qual foi utilizado o BMQ, 46,7\% dos participantes aderiram ao uso de antidiabéticos orais ${ }^{13}$. 
Para o domínio "Regime", 75,5\% não apresentaram barreiras neste estudo. Dos que apresentaram barreiras, todos relataram ter esquecido de tomar, pelo menos, uma dose durante a última semana. No domínio relacionado às "Crenças", que diz respeito à presença ou não de medicamentos que não funcionam bem, ou que causam algum problema para o paciente, $91,1 \%$ dos pacientes não apresentaram barreiras. Analisando-se quais os domínios que influenciam no escore total, constatou-se que o domínio regime explica $50 \%$ da variabilidade do BMQ total. Assim, o relato de falha de doses pode ser considerado importante no resultado final de adesão ao tratamento.

Outro estudo apontou que os $82 \%$ dos participantes com diabetes acreditavam que os medicamentos prescritos melhoram os sintomas da doença ${ }^{14}$. Esses indivíduos apresentaram maiores taxas de adesão ao tratamento medicamentoso do que aqueles que se apresentaram descrentes em relação ao medicamento. Isso mostra a importância do conhecimento dos pacientes em relação ao tratamento, que é adquirido, muitas vezes, através da equipe de saúde.

No domínio de "Recordação", que se refere a presença de um esquema de múltiplas doses diárias e o relato de dificuldades para lembrar de tomar a medicação, 100\% obtiveram um escore compatível com não adesão, pois todos os pacientes são tratados com múltiplas doses de insulina diárias. Um estudo realizado em Pernambuco em 2008 mostrou que $50 \%$ dos participantes relataram "esquecer de tomar" seus medicamentos ${ }^{15}$.

O clássico estudo prospectivo Diabetes Control and Complications Trial (DCCT) demonstrou que o tratamento intensivo do DM1, com a aplicação de múltiplas doses de insulina com diferentes tipos de ação, é eficaz em reduzir a frequência de complicações crônicas do DM ${ }^{12}$.

O tempo de diagnóstico também é um fator importante na adesão ao tratamento medicamentoso. A OMS aponta relação inversa entre o tempo de diagnóstico do diabetes e a adesão dos pacientes à terapia medicamentosa, isto é, indivíduos que possuem o diagnóstico de diabetes a mais tempo tendem a apresentar menores taxas de adesão ao tratamento ${ }^{16}$. Dos participantes deste estudo, 73,3\% possuíam tempo de diagnóstico igual ou superior a 5 anos, desses indivíduos cerca da metade apresentou resultado no BMQ total compatível com baixa adesão. Estes dados são preocupantes, visto que os riscos de complicações crônicas tendem a aumentar com o tempo da doença.

Quanto a avaliação das capacidades motivacionais e emocionais para o autocuidado, 75,5\% dos participantes apresentaram déficit moderado de motivação, e apenas 6,7\% comportamento motivacional positivo. Um estudo realizado em Fortaleza com 100 idosos apontou que 31\% dos idosos apresentaram déficit de motivação para o autocuidado e 69\% demonstraram média motivação para o autocuidado na subescala III do ECDAC ${ }^{17}$.

Os motivos pelos quais os adolescentes e adultos jovens não apresentaram motivação para autocuidado podem estar relacionados com as recomendações para um bom controle da doença, como a prática de exercícios físicos e hábitos alimentares saudáveis, visto que esses foram os itens que menos pontuaram na subescala III do ECDAC. Isso ressalta a importância da educação para a saúde providenciada pela equipe multidisciplinar que os acompanha, ajudando-os a compreender melhor a doença e o tratamento.

A motivação para o autocuidado é fundamental para que o indivíduo tenha uma boa adesão ao tratamento e, consequentemente, um bom controle glicêmico. Em relação ao tratamento, poucos pacientes relataram se sentir incomodados com as aplicações da insulina. No entanto, um dos problemas mais citados foi a dificuldade de lembrar de fazer as aplicações de insulina. Fatores como o esquema de múltiplas doses de insulina e o esquecimento de administração de alguma dose contribuíram para os resultados do BMQ de menor adesão à terapia medicamentosa.

Traçar um perfil socioeconômico e investigar os fatores que mais influenciam os adolescentes e adultos jovens na motivação do autocuidado e, consequentemente, na adesão ao tratamento da DM1, possibilita o planejamento de ações de promoção e prevenção da saúde. Os resultados obtidos nesta pesquisa mostraram uma maior prevalência do sexo masculino, de raça branca e de estudantes. Além disso, pouco mais da metade da população investigada provavelmente adere ao tratamento medicamentoso. Contudo, uma grande parcela da amostra apresentou provável baixa adesão. Os aspectos que mais contribuíram para a baixa adesão referem-se ao esquema de múltiplas doses de insulina e as dificuldades de lembrar da medicação. Quanto a motivação do autocuidado, a maioria dos participantes possuíram déficit moderado de motivação, devido a recomendações para ter uma vida saudável, como manter a dieta e a prática de atividade física.

As principais limitações do estudo foram relacionadas com a coleta de dados em um período reduzido e a inclusão de apenas pacientes que estavam frequentando o Centro de Referência com regularidade mínima de 6 meses, não tendo sido buscado os pacientes faltosos, que, provavelmente, teriam apresentado uma menor taxa de adesão ao tratamento.

Destaca-se, por fim, a importância dos profissionais de saúde no desenvolvimento de ações de promoção e prevenção em saúde, de maneira a capacitar os pacientes com diabetes a respeito da doença, para que sejam feitas 
escolhas saudáveis em seu cotidiano, com vistas à melhoria do controle da doença e da qualidade de vida dessa população.

\section{REFERÊNCIAS}

1. Leal DT, Fialho FA, Dias IMAV, Nascimento L, Arruada WC. Diabetes na infância e adolescência: o enfrentamento da doença no cotidiano da família. HU Rev. 2009;(35)4:288-95.

2. Calliari LEP, Monte O. Abordagem do diabetes melito na primeira infância. Arq Bras Endocrinol Metab. 2008;52(2):243-9. https://doi. org/10.1590/S0004-27302008000200011

3. Santos JR, Enumo SRF. Adolescentes com diabetes mellitus tipo 1: seu cotidiano e enfrentamento da doença. Psicol Reflex Crít. 2003; 16(2):411-25. https://doi.org/10.1590/S0102-79722003000200021

4. Minanni CA, Ferreira AB, Sant'Anna MJC, Coates V. Abordagem integral do adolescente com diabetes. Adolesc Saúde. 2010;7(1): 45-52.

5. Silverstein J, Klingensmith G, Copeland K, Plotnick L, Kaufman F, Laffel L, Deeb L, Grey M, Anderson B, Holzmeister LA, Clark N; American Diabetes Association. Care of children and adolescents with type 1 Diabetes. Diabetes Care. 2005 Jan;28(1):186-212. https://doi.org/10.2337/diacare.28.1.186

6. International Diabetes Federation (IDF). IDF diabetes atlas [Internet]. 8ạ ed. 2017 [capturado 2018 Ago 29]. Disponível em: https://www. idf.org/e-library/epidemiology-research/diabetes-atlas.html

7. Ben AJ, Neumann CR, Mengue SS. Teste de Morisky-Green e Brief Medication Questionnaire para avaliar adesão a medicamentos. Rev Saúde Pública. 2012;46(2):279-89. https://doi.org/10.1590/S003489102012005000013

8. Lima LAP, Weffort, VRS, Borges MF. Avaliação da qualidade de vida de crianças com diabetes mellitus tipo 1. Ciênc Cuid Saúde. 2011;10(1):127-33.

9. Marques RMB, Fornés NS, Stringhini MLF. Fatores socioeconômicos, demográficos, nutricionais e de atividade física no controle glicêmico de adolescentes portadores de diabetes melito tipo 1. Arq Bras Endocrinol Metab. 2011;55(3):194-202. https://doi.org/10.1590/ S0004-27302011000300004

10. Diretrizes da Sociedade Brasileira de Diabetes 2017-2018 [Internet]. São Paulo: Editora Clannad; 2017 [capturado 2018 Ago 29]. Disponível em: https://www.diabetes.org.br/profissionais/ images/2017/diretrizes/diretrizes-sbd-2017-2018.pdf

11. Andrade CJN. Influência dos fatores socioeconômicos e afetivoemocionais no controle glicêmico de crianças com diabetes mellitus tipo 1 [dissertação]. Salvador: Universidade Federal da Bahia. Instituto de Ciências da Saúde; 2013.

12. Diretrizes da Sociedade Brasileira de Diabetes. Tratamento e acompanhamento do diabetes mellitus. Aspectos clínicos e laboratoriais da hemoglobina glicada. São Paulo: Sociedade Brasileira de Diabetes; 2016.

13. Istilli PT. Adesão ao agente antidiabético oral de pessoas com Diabetes mellitus: uso do Brief Medication Questionnaire [dissertação]. Ribeirão Preto: Universidade de São Paulo. Escola de Enfermagem de Ribeirão Preto; 2014.

14. Grant RW, Devita NG, Singer DE, Meigs JB. Polypharmacy and medication adherence in patients with type 2 diabetes. Diabetes Care. 2003;26(5):1408-12. https://doi.org/10.2337/ diacare.26.5.1408

15. Bezerra DS, Silva AS, Carvalho ALM. Avaliação das características dos usuários com hipertensão arterial e/ou diabetes mellitus em uma Unidade de Saúde Pública, no município de Jaboatão dos Guararapes-PE, Brasil. Rev Ciênc Farm Básica Apl. 2009;30(1): 69-73.

16. Organização Mundial da Saúde. Cuidados inovadores para as condições crônicas: componentes estruturais de ação: relatório mundial. Brasília: OMS; 2003.

17. Marques MB, Silva MJ, Coutinho JFV, Lopes MVO. Avaliação da competência de idosos diabéticos para o autocuidado. Rev Esc Enferm USP. 2013;47(2):415-20. https://doi.org/10.1590/S008062342013000200020 